\title{
Performance evaluation of an IEEE802.15.4 standard based Wireless Sensor Network in Mars exploration scenario
}

\author{
Renato Pucci ${ }^{\#}$, Luca Simone Ronga ${ }^{\#}$, Enrico Del Re ${ }^{\#}$, Demis Boschetti ${ }^{*}$ \\ " Dipartimento di Elettronica e Telecomunicaizoni \\ University of Florence \\ Florence, Italy \\ renato.pucci@unifi.it, luca.ronga@cnit.it, enrico.delre@unifi.it \\ * B.U.O.O.S \\ Thales Alenia Space Italia \\ Turin, Italy \\ demis.boschetti@thalesaleniaspace.com
}

\begin{abstract}
Born as data networks for personal areas (WPAN), wireless sensor networks (WSN) have rapidly gained a growing importance in many other contexts. One of the most interesting and innovative field of application of WSN is represented by the planetary exploration context; taking into account the actual scopes of NASA and ESA, the aim of this paper is to present the performance evaluation of an IEEE802.15.4 standard based network in Mars exploration context. Thanks to an analysis of the Martian geomorphologic, atmospheric and eolian features, a characterization of five most representative frequency channels will be given. Considering such analysis, an evaluation of the performance of an IEEE802.15.4 standard based network is obtained, performing simulation processes with Simulink ${ }^{\circledR}$ and OMNET++.
\end{abstract}

WSN; Planetary exploration; IEEE802.15.4; ZigBee; Mars; Wireless Sensor Network.

\section{INTRODUCTION}

The interest for wireless communication in space missions have been growing-up in the last years, driven on one side by the complexity on new space systems and supported by the technological trend for ground commercial utilization, that makes available standards and products based on RF. Potential space applications for RF wireless systems are numerous, like planetary surface exploration, intra-satellite devices communication, extra-vehicular operations.

About planetary exploration missions, after the failure of the Beagle II Mars mission, the research community has focused the attention on redundant systems, like WSN, in which many small sensors are deployed, where, if some sensors should fail, the mission would not. The IEEE 802.15.4 standard (ZigBee) provides low cost and low power connectivity for WSN devices that need a monthly or year's duration of battery, with low datarate and small dimensions. In order to valuate ZigBee usability in planetary exploration context, a set of possible scenario is studied, referring to a realistic scenario of Mars. Therefore, thanks also to the recent information given by Phoenix Mission, in this paper different Martian radio frequency channel models are used; such models take into account all Martian features, like Tropospheric effects, clouds, wind, snow, gaseous attenuation and dust storm effect. The knowledge about such channels allow to investigate system transmission and network performance, thanks to simulation process.

\section{RELATED WORK}

In order to obtain a characterization of the channels used by WSN and its performance, we follow the block diagram shown in Figure 1.

Analyzing complex input response and fading statistics, thanks to their statistical, we obtain a quality and data rate estimation of the channel; therefore, such parameters are used together with PHY and MAC specifications of the standard to execute simulations processes in Simulink ${ }^{\circledR}$ (to obtain Bit Error Rate (BER) performance of an OQPSK transmission system) and OMNET++ (to obtain an estimate of the network performances). 


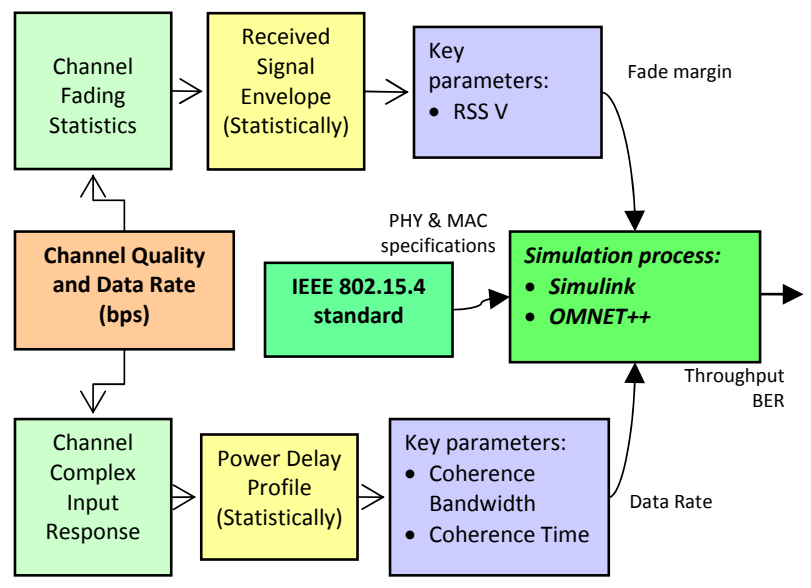

Figure 1. Analysis process used to obtain WSN performance in planetary exploration context.

\section{MARTIAN CHANNEL CHARACTERIZATION}

\section{A. Coherence Bandwidth and Time}

Due to diffraction, reflection and scattering effects caused by presence of obstacles near the propagation path, the receiver collects not only one signal, but a signal multiplicity composed by direct signal (LOS, "Line Of Sight") and its delayed replicas (Non-LOS); variation due to multipath occurs over very short distances and they are called "multipath fading". An important characteristic of a multipath channel is the time delay spread, that equals the time delay between the arrival of the first received signal component (LOS or multipath) and the last received signal component associated with a single transmitted pulse.

Coherence bandwidth is the approximate maximum bandwidth or frequency interval over which two frequencies of a signal are likely to experience comparable or correlated amplitude fading. Coherence time is the time duration over which the channel impulse response is essentially invariant.

If the multipath time-delay spread equals $\tau$ seconds and the frequency Doppler $\mathrm{f}_{\mathrm{D}}[\mathrm{Hz}]$, then the coherence bandwidth $\mathrm{W}_{\mathrm{C}}$ $[\mathrm{Hz}]$ and coherence time are given approximately by the equations:

$$
\begin{gathered}
W_{c} \approx \frac{1}{2 \pi \cdot \tau} \\
T_{c} \approx \sqrt{\frac{9}{16 \pi \cdot f_{D}^{2}}}=\frac{0.423}{f_{D}}
\end{gathered}
$$

Hence, we can summarize for:

\begin{tabular}{|c|c|c|c|}
\hline$T_{S}<<T_{c}$ & $\begin{array}{c}\text { approx. non-time- } \\
\text { variant channel } \\
\text { (Doppler effect is } \\
\text { negligible) }\end{array}$ & $B<W_{c}$ & $\begin{array}{c}\text { approx. flat } \\
\text { channel } \\
\text { (Doppler effect is } \\
\text { negligible) }\end{array}$ \\
\hline$T_{S} \geq T_{c}$ & $\begin{array}{c}\text { time-variant } \\
\text { channel } \\
\text { (Doppler effect isn't } \\
\text { negligible) }\end{array}$ & $B>W_{c}$ & $\begin{array}{c}\text { frequency } \\
\text { selective } \\
\text { channel }\end{array}$ \\
\hline
\end{tabular}
results:

Considering the context that we are studying, the channel

- Non-frequency-selecting, since it results $B<W_{c}$.

- Time-invariant, since it results $T_{S}<T_{C \text {; }}$ thinking to install a sensor on the exploring rover (able to move), Doppler effect is negligible due to very low speed in question.

\section{B. Received Signal Envelope}

Considering the planetary exploration scenario of Mars, there are several features affecting the propagation of radio wave, like presence of clouds, snowfalls, temperatures, gaseous composition of the Martian atmosphere (different from the Earth's one), presence of obstacles (rocks and craters) and possible occurrence of dust-storms. Among them, last two features offer the major contributions to the radio wave attenuation and, furthermore, such contributions vary according to Martian region of interest.

Taking into account different surface morphology scenarios (see Figure 2) in terms of rock dimension and density, we consider two different channel mode:

- "Normal" channel (Low-Medium rock density and dimension),

- "Rocky" channel (High rock density and dimension).

In particular, considering the possible wave-propagation paths (LOS and NLOS) and the multipath components, "Normal" channel can be described statistically as a Ricean channel, with a Rice Factor $\mathrm{k}=10$ (ratio between LOS and NLOS components powers) and a probability density function given by:

$$
p(E)=\frac{E}{\sigma^{2}} e^{-\frac{E^{2}+A^{2}}{2 \sigma^{2}}} I_{0}\left(\frac{E A}{\sigma^{2}}\right)
$$

where the parameter A denotes the peak amplitude of the dominant signal, $\mathrm{I}_{0}(\bullet)$ is the modified Bessel function of the first kind and zero-order and $\sigma^{2}$ is the time-average power of the received signal. 


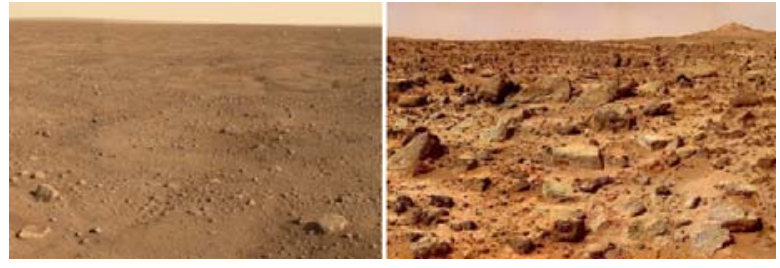

Figure 2. Different rock density regions of Mars, made by "Phoenix" and "Viking1" landers.

"Rocky" terrain scenario is characterized thanks to the frequency distributions obtained from data collected by Viking 1 and 2 landers; such distributions give information about dimensions and number of rocks per $\mathrm{m}^{2}$ of Martian rocky landing sites.

Taking into account such information and comparing sensors and rocks dimensions, "Rocky" channel can be described statistically as a Rayleigh channel, with a probability density function given by:

$$
p(E)=\frac{E}{\sigma^{2}} e^{-\frac{E}{2 \sigma^{2}}} \quad E \geq 0
$$

Dust-storms occur primarily in the south hemisphere of Mars, but sometimes they can cover the whole planet. Since they differ in dust particle density (from $N_{T}=1 \times 10^{7} \mathrm{~m}^{-3}$ to $N_{T}=8 \times 10^{7} \mathrm{~m}^{-3}$ ) [1] and wind strength (from 2 to $28 \mathrm{~m} / \mathrm{s}$ ), we consider three different channels:

- "Faint Dust-Storm" channel (Low particle density, faint wind, $\bar{r}=1 \mu \mathrm{m}$ )

- "Strong Dust-Storm” channel (Medium particle density, strong wind, $\bar{r}=10 \mu \mathrm{m}$ )

- "Heavy Dust-Storm" channel (High particle density, heavy wind, $\bar{r}=20 \mu \mathrm{m}$ )

Referring to [2], the attenuation caused by a sand-storm could be obtained by:

$$
A(\lambda)=\frac{1.029 \times 10^{6} \varepsilon^{\prime \prime}}{\lambda \cdot\left[\left(\varepsilon^{\prime}+2\right)^{2}+\varepsilon^{\prime \prime 2}\right]} N_{T} \bar{r}^{3}[\mathrm{~dB} / \mathrm{km}]
$$

where $\lambda$ is wavelength in meters, $\mathrm{N}_{\mathrm{T}}$ is the total particle density in $\# / \mathrm{m}^{3}, \varepsilon^{\mathrm{I}}$ and $\varepsilon^{\mathrm{II}}$ are the real and imaginary part of the dielectric permittivity index and $\bar{r}$ is the mean particle radius in meters, obtained through an integration over all sizes of particles in the normalized particle number density N(r). At the present time there aren't accurate measurement of the mean particle radius, but it can be considered included in $[1 \mu \mathrm{m}$; $20 \mu \mathrm{m}]$.

\section{BER performances}

For everyone of the five channel model above described, we obtain the BER performances of OQPSK modulation (used in IEEE 802.15.4 standard [3]) for different distances of transmission. The results shown in Figure 3 and 4 are obtained simulating the transmission system with a Simulink ${ }^{\circledR}$ based model, varying parameters of "Channel" block according to different channel simulation.

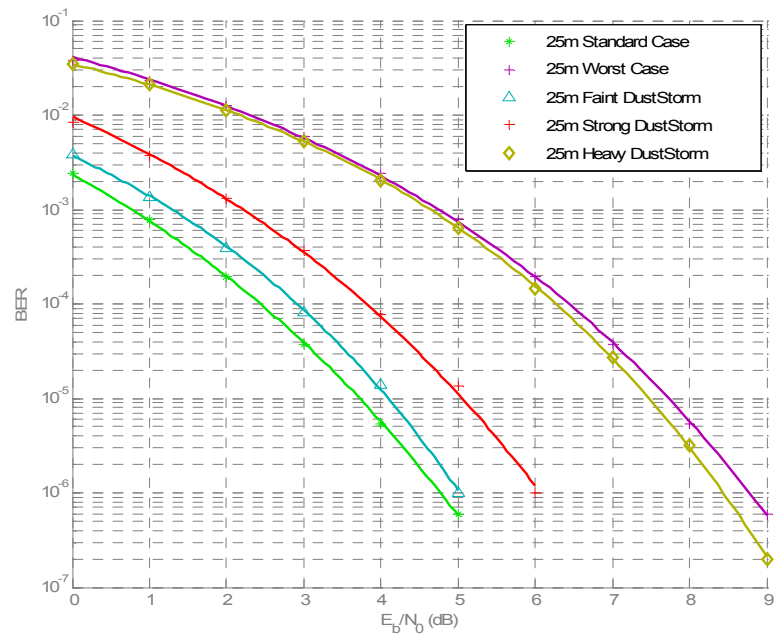

Figure 3. BER trends comparison among different channels, considering a $25 \mathrm{~m}$ distance transmission and OQPSK modulation.

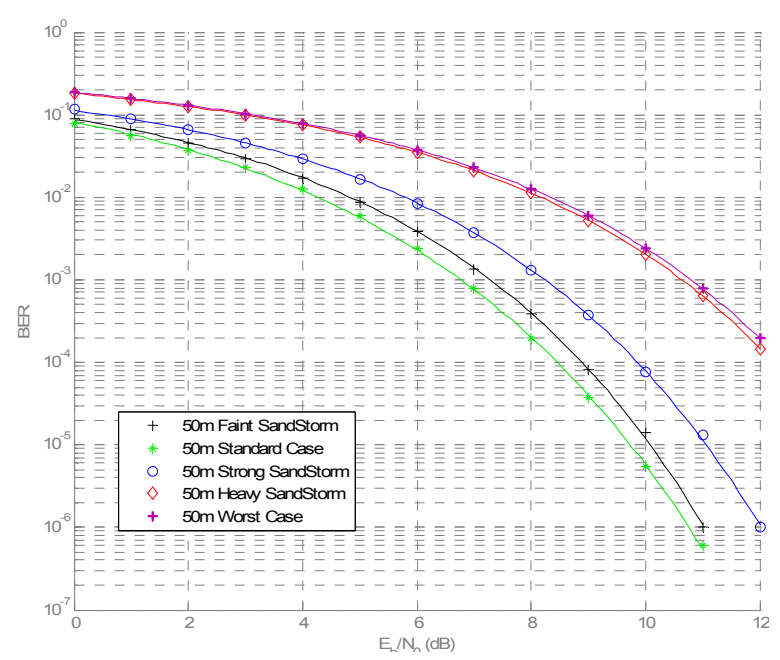

Figure 4. BER trends comparison among different channels, considering a $50 \mathrm{~m}$ distance transmission and OQPSK modulation.

\section{OMNET++ SIMULATIONS}

\section{A. Used Model}

Thanks to OMNET++, an open source discrete event simulation system, we realized a transmission system of a IEEE 802.15.4 network, formed by a mobile rover and 40 sensors, randomly deployed in a $100 \mathrm{~m}^{2}$ square area. Every sensor is defined as a compound element, since the architecture of every sensor is composed by modules(see Figure 5); "Physic" block is used to create, maintain and delete the transmission links, "MAC" block checks the MAC header of every packet, "AODV" block represent the routing 
algorithm, "Application" is used to described the type of the collected data (i.e. temperature, pressure, seismic activity, etc), "Battery" block simulate the battery life of the sensor (it decreases for every sent message); "Mobility" block defines the direction and the velocity of the rover.

Taking into account the possibility of sensor damage or transmission inability caused by a bad deployment, we consider a sensor loss equals to $10 \%$.
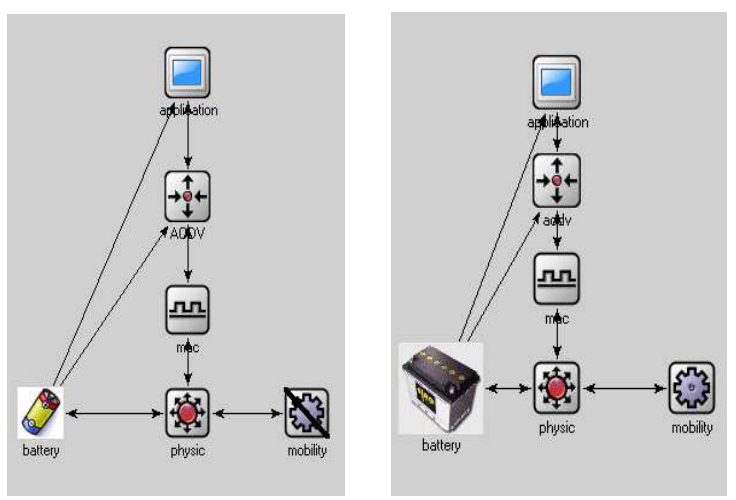

Figure 5. Sensor (left side) and rover (right side) architecture in OMNET++.

Referring to IEEE 802.15.4 physical specifications for OQPSK modulated transmission at $2.4 \mathrm{GHz}$, we consider an non-coded Bit Error Probability given by:

$$
\begin{aligned}
& P_{e}\left(S N R_{20 m, d b}, d\right)= \\
& =\frac{1}{2} \operatorname{erfc}\left(\sqrt{\left[S N R_{20 m, d b}+L_{20 m, d b}-L(d)_{d b}\right]_{l i n}}\right)
\end{aligned}
$$

where $S N R_{20 m, d b}$ is the reference SNR term at the receiver placed at $20 \mathrm{~m}$ from the transmitter (in $\mathrm{dB}$ ) and the []$_{\text {lin }}$ operator represents the linear conversion of the argument. The pathloss terms are obtained considering:

$$
P_{R X}=\frac{P_{T X}}{L(d)}
$$

where

$$
\begin{aligned}
& L(d)=\left(\frac{4 \pi \cdot d}{\lambda}\right)^{3} \\
& L_{20 m}=L(20)
\end{aligned}
$$

Since the reference scenario is a terrain with a medium/high density of rocks, we consider a $3^{\text {rd }}$ order exponent for the Path Loss as experimental approximation for terrains with numerous scatterers. Imposing the Bit Error Probability maximum equals to $4.8 \cdot 10^{-5}$, we obtain that 20 meters transmissions are permitted at least.

\section{B. Simulation Results}

In order to evaluate the variation of the network performance during a $24 \mathrm{~h}$-transmission, we establish that on time $\mathrm{t}=6 \mathrm{~h}$ of the simulation, a $12 \mathrm{~h}$-sandstorm occurs, as shown in Figure 6. The intensity of the sandstorm depends on $\mathrm{N}_{\mathrm{T}}$ and the attenuation is calculated with equation (5).

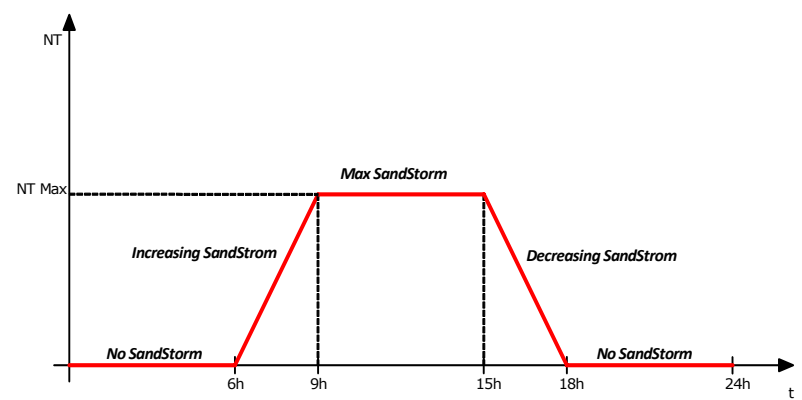

Figure 6. Simulation time sequence: occurrence of a sandstorm.
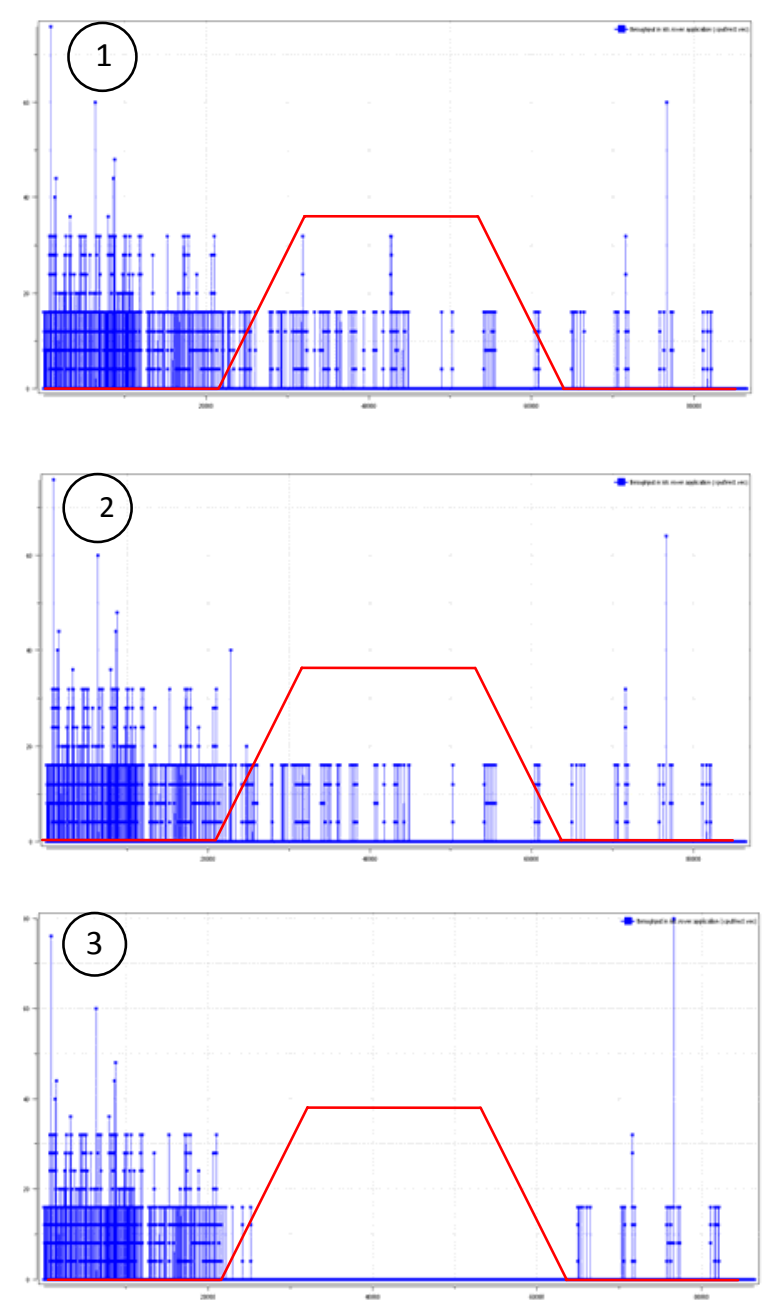

Figure 7. Throughput measured by the Rover in case of : "Faint" sandstorm (1), "Strong" sandstorm (2), "Heavy" sandstorm (3). 
In line with the above mentioned Martian channel in presence of dust storm, we consider three different cases of simulation. Figure 7 shows the simulation results; a clear throughput performance degradation is appreciable, according to the increase of the sandstorm intensity.

In case of "faint" and "strong" sandstorm throughput performance makes lower, but network still works. Nevertheless for an "heavy" sandstorm no transmission is possible and networks turns off.

\section{CONCLUSIONS}

In this paper we consider the opportunity to use an IEEE 802.15.4 standard based network in Martian planetary exploration context. Thanks to an evaluation of the main features of Mars, we define five different channels that model the five most common propagation contexts. In order to obtain the channel data rate and availability, a capability analysis is performed, considering the power delay profile, the received signal envelope and the measure of channel RF traffic. For the mentioned channels, we evaluate BER and throughput performances of a WSN, performing 24h-long simulation campaigns.

Considering a network formed by a mobile rover and 40 sensors, we results obtained from simulations demonstrate that an IEEE802.15.4 based WSN can be used in planetary exploration context. Such WSN works pretty well, also in case of transmission within terrains with high density of rocks.

In the case of sand storms occurrence, network performances degrade proportional with the storm intensity and dust particles dimensions. For faint dust storm, WSN works well; in case of heavy dust storm, WSN performances become critical. Nevertheless the WSN is able to react positively after the cessation of the perturbation.

The results shown in this paper, demonstrate that WSN should be used in future mission of planetary exploration; a test campaign should follow in order to validate simulated and predicted data.

\section{ACKNOWLEDGMENT}

This paper contains OMNETT++ simulations results made by Francesca Paradiso and Jacopo Pesci.

\section{REFERENCES}

[1] J. Goldhirsh, "A parameter review and assessment of attenuation and backscatter properties associated with dust storms over desert regions in the frequency range of 1 to $10 \mathrm{GHz}$ ", IEEE Trans. Ant. Propaga., AP$30,1121,1982$.

[2] T.S. Chu, "Effects of sandstorms on microwave propagation", Bell Syst. Tech. J., 1979.

[3] IEEE Std 802.15.4-2006, "Part15.4: Wireless Medium Access Control (MAC) and Physical Layer (PHY) Specifications for Low-Rate Wireless Personal Area Networks (WPANs)". 